\title{
Correlación citológica e histológica de las imágenes colposcópicas en el diagnóstico de neoplasia cervical
}

\author{
Carlos Alberto Díaz Rodríguez*; Miguel Aragón Sánchez**
}

\begin{abstract}
RESUMEN: Se revisaron las historias clínicas de las pacientes de la clínica de colposcopia, que ingresaron en el estudlo entre los años de 1988-1989. Se estudiaron las imágenes colposcópicas y se correlacionaron con los diferentes estudios: Citología, resultado de patología en la biopsia $y$ en la pieza final. El estudio incluye 400 casos.

La sensibilidad de la citología corresponde al $93 \%$, especificidad el $42 \%$ y falsos negativos del $7 \%$. La citología fue negativa en $4 \%$ de los casos con NIC III y en $9 \%$ de casos con Ca. invasivo.

La citología A2 con atipias severas se correlacionó con casos de NIC II - III en $21 \%$ de las pacientes. La Citología A5 presenta una frecuencia de falsos negativos para Ca Invasivo de $\mathbf{4 4} \%$ y la citología A4-A5, presenta tasas de falsos negativos frente a cérvix del $\mathbf{7 6} \%$.

El porcentaje de colposcopia insatisfactoria es del $34 \%$, siendo en mayores de 40 años de $52 \%$. Las pacientes con lesión endocervical presentan una tasa de colposcopia insatisfactoria del $67 \%$.

La sensibilidad de la colposcopia en nuestra institución es del $88 \%$ con una especificidad del $39 \%$ y unos falsos negativos del $12 \%$, frente a la citología.

Frente al resultado de patología, la colposcopia tiene una sensibilidad del $89 \%$, una especificidad del $64 \%$ y una tasa de falsos negativos del $11 \%$.

En el estudio de lesión por papilomavirus, la citología solo da una sensibilidad del $\mathbf{1 2} \%$, con un índice de falsos negativos del $88 \%$.

La colposcopia frente a la lesión por condiloma da una sensibilidad del $63 \%$ y unos falsos negativos del $37 \%$.

En los pacientes con biopsia que refería compromiso glandular, la anatomía patológica final no reveló en ningún caso invasión.

La correlación de imágenes colposcópicas se establece así:

Cervicitis: zona de transformación típica, epitelio acetoblanco.
\end{abstract}

NIC: Leucoplasia, punteado y mosaico.

Microinvasión: Leucoplasia y vasos IV.

Ca. Invasivo: Leucoplasia, punteado, vasos IV y V, proliferación carcinomatosa y úlcera.

La frecuencia de lesión encontrada en los dos años son:

Lesión benigna: $29.5 \%$, NIC I: $11 \%$, NIC II, 9.5\%, NIC III; 37\%, microinvasivo: $1 \%$ y Ca. Invasivo: $12 \%$.

PALABRAS CLAVE: NIC: Neoplasia Cervical Intraepitelial

SUMMARY: The study group consisted of $\mathbf{4 0 0}$ patients from clinical colposcopy. The colposcopic features correlations with citology, biopsy and worst pathologic result on cone or Hysterectomy.

The sensitivity rate of citology was $93 \%$, specificity rate of $42 \%$, and false-segative rate was $7 \%$. The citology was negative in $4 \%$ of cases with C.I.N III (Cervical Intraepithelial Neoplasia) and $9 \%$ with invasive cancer cases.

The $\mathbf{A} 2$ citology and severe atypias cases findings $21 \%$ of C.I.N.II-III. The unsatisfactory colposcopy rate was $34 \%$, and 40 years old patients was $52 \%$.

In patients with endocervical lesions findings unsatisfactory colposcopy rates of $67 \%$.

The sensitivity rate of colposcopy (compared with citology) was of $88 \%$, specificity rate of $39 \%$, and false-negative rate of $12 \%$.

The sensitivity rate of colposcopy (compared with final pathology) was $89 \%$, specificity rate of $64 \%$, and false-negative rate was $11 \%$.

In the study for papilomavirus lesions, the sensivity rate of citology only was of $63 \%$, and false-negative rates of $37 \%$.

The colposcopic images were correlated with lesions revealing following patterns:

Cervicitis: Atypical transformation zone, aceto-White epithelium.

C.I.N.: White epithelium (leukoplakia), punctation, mosaic. microinvasive: Leukoplakia, atypical vessels (IV). Invasive cancer: Leukoplakia, punctation, atypical vessels (IV, V), carcinomatose proliferation, and ulcus.

The lesions frecuency in the study were:

Beningns lesions: $29.5 \%$, C.I.N. I:11\%, C.I.N II:9.5\%, C.I.N III:37\%, microinvasive: 1\%, invasive cancer; $12 \%$.

KEY WORDS: CIN: Cervical Intraepitelial Neoplasia

\section{Introducción}

Múltiples estudios dan una tasa de falsos negativos de la citología que oscila entre el $1.8 \%(13)$ al $40 \%(10,15,23)$.

\section{BIBLIOGRAFIA (Comentario Dr. Hernando Gaitán)}

* Residente III -Ginecología y Obstetricia- Universidad Nacional

** Profesor Asistente -Ginecología y Obstetricia- Jefe Sección de Oncología, Departamento de Obstetricia y Ginecología, Universidad Nacional de Colombia Bogotá.
Según el grado de lesión, encontramos en varios autores un índice de negatividad de la citología así:
Biopsia

Nic I

II

III

CIS
Citología

(31)

$43.4 \%$

22.0

13.7
$20-45 \%$ 
Algunos autores refieren que los falsos negativos de la citología disminuyen con lesión más avanzada (1), aunque otros reportan que la citología es menos sensible para detec$\operatorname{tar} \mathrm{Ca}$. invasivo (10). Así en carcinoma invasivo, se encuentran falsos negativos del 6 al 55\% (23).

En busca de disminuir la tasa de falsos negativos de la citología, se han ideado métodos como el aspirado del orificio endocervical externo y raspado exocervical exhaustivo, encontrando que la tasa de falsos negativos con estos métodos es menor:

\section{Falsos negativos \\ Aspirado del orificio externo $4-17 \%$ \\ Raspado cervical 6-18\% (23)}

Es de esperar que el Scalping endocervical y el raspado vigorozo de la zona de transformación, mejoren la tasa de positividad de la citología. Frente a la colposcopia, los falsos negativos de la citología oscilan en los diferentes estudios en un 10.3 a un $13 \%$ (13).

Según Javaheri (26), la correspondencia entre la citología y los grados colposcópicos, es:

\begin{tabular}{llr} 
Citología & \multicolumn{2}{c}{ \% Grado Colposcópico } \\
A2 & $13 \%$ & Grado III \\
& $41 \%$ & Grado I - II \\
A3 & $48 \%$ & Grado III \\
& $36 \%$ & Grado I - II \\
A4 & $86 \%$ & Grado III \\
& $3 \%$ & Ca. Invasivo
\end{tabular}

Se encuentran informes del grado de lesión histológica en relación con la clase de la citología, así: (38):

Citología
A1
A2 (\&)
A3
A4
A5

Dx Histológico
$(-)$
$(-)$
Nic I-II
Nic III
Invasión

(\&): Según los estudios de Noumoff y Jones $(36,37)$, la citología A2 con atipias severas, puede corresponder en un $25-29 \%$ a NIC. Otros refieren inclusive tasas tan altas como del $75 \%(36)$.

En relación con la colposcopia, se encuentran frecuencias en los diferentes estudios que oscilan entre el 4 y el 57\%, de insatisfactoria. Todos los autores están de acuerdo con que la tasa de colposcopia insatisfactoria aumenta con la edad, y es mayor de $15 \%$ en mujeres mayores de $40-45$ años $(5,13,14,18)$. Hatch, encuentra inclusive una tasa de $75-78 \%$ de colposcopia insatisfactoria en pacientes mayores de 45 años (3).

Esto se debe a la tendencia a la endopía y la migración de epitelio por los cambios hormonales, de acuerdo con lo establecido por los estudios de Hamperl (39).

Los falsos negativos de la colposcopia, oscilan según los diferentes autores así:

Comparando con la biopsia: del 0.5 al $30 \%(12,30)$

Comparada con el cono: del 5.6 al 15\% (12).
La relación entre el grado de lesión colposcópico (CP) y el grado histológico (AP) es dado por algunos autores así:

\begin{tabular}{lrcc} 
& \multicolumn{3}{c}{ Estudio } \\
CP $=$ Ap & $\mathbf{3 0}$ & $\mathbf{2 6}$ & $\mathbf{1 4}$ \\
Cp $>$ Ap & $41-67 \%$ & $91 \%$ & $85.0 \%$ \\
Cp $>$ Ap & $6-26 \%$ & $5 \%$ & 11.7 \\
& $3-20 \%$ & $1.8 \%$ & $3.3 \%$
\end{tabular}

De todas maneras todos los estudios dan una correlación entre colposcopia y lesión histológica que va del $78 \%(34)$ al $92 \%(31)$.

Las imágenes colposcópicas se relacionan directamente con la lesión, y en la tabla No. 1, se dan relaciones de imágenes y resultado histopatológico según varios autores.

Podemos resumir lo encontrado en la litertura de las imágenes colposcópicas para las diferentes patologías así:

Lesión

\section{Imagen Colposcópica}

NIC:

Leucoplasia, punteado, mosaico: (7) $\mathrm{CHOO}$, $(9,14)$ Stafl, (15) Disaia, Creasman, (19) Mestwert, (24) Hinselman, koldstad.

Microinvasivo: Leucoplasia, punteado y mosaico: (7), (11), (24), vasos atípicos en superficie y bordes irregulares (11) que proliferan en grandes campos de Mosaico. $(24,8)$.

Ca invasivo: Vasos atípicos IV-V: $(1,2,9,12,14,15$, $16,19,24,25)$ Leucoplasia gruesa $(16,19,21$, 22) Ulcera $(9,16)$ Erosión $(5,12,19)$.

\section{Material y métodos}

Se revisaron las historias de las pacientes que ingresaron al estudio de colposcopia entre los años de 1988-1989, en el Instituto Materno Infantil de Bogotá.

Se estudiaron las colposcopias y biopsias de cuello de dichas pacientes y su tratamiento posterior, estudiando además el resultado de anatomía patológica definitivo en la pieza quirúrgica.

Las pacientes que no tienen estudio histopatológico adecuado se excluyen del estudio. Es de anotar que todas las biopsias aquí analizadas, son biopsias dirigidas bajo colposcopia.

Se tomaron los resultados de la encuesta y se colocan en una tabla de 4 casillas $(2 \times 2)$, para establecer los valores de significancia de las diferentes variables a cruzar.

Se usará en cada caso la prueba del $\mathrm{Ji}^{2}$ para significancia estadística. La frecuencia de colposcopia insatisfactoria, así como la frecuencia de lesión endocervical, se analizarán en forma descriptiva.

Se cruzarán de acuerdo con los anteriores parámetros, las siguientes variables:
-Citología frente a biopsia o Anatomía patológica definitiva.
-Colposcopia frente a citología.
- Colposcopia frente a Anatomía Patológica para lesión por condiloma. 
Tabla No. 1:

FRECUENCIA DE IMAGENES COLPOSCOPICAS Y SU RELACION CON DIAGNOSTICO HISTOPATOLOGICO, SEGUN LOS DIFERENTES AUTORES

\begin{tabular}{|lcccl|}
\hline \multicolumn{4}{|c|}{ Imágenes Colposcópicas } \\
\hline LESION & $\begin{array}{c}\text { Epitelio } \\
\text { Acetoblanco }\end{array}$ & $\begin{array}{c}\text { Leuco- } \\
\text { Plasia }\end{array}$ & $\begin{array}{c}\text { Base } \\
\text { (Punteado) }\end{array}$ & Mosaico \\
\hline $\begin{array}{l}\text { Cervicitis } \\
\text { Nic I }\end{array}$ & $(20) 100 \%$ & & $(24) 1.2 \%$ & $(24) 0.6 \%$ \\
Nic II & & & $(21)$ & $(24) 0.7 \%$ \\
Nic III & & $(24) 61.1 \%$ & $(19)(21) 21.1 \%$ \\
& & & $(24) 28 \%$ \\
Microinva- & & $(19)(20)$ & $(24) 45.5 \%$ \\
sivo. & & $(7) .5 \%$ & $(7)$ \\
Ca. Inva- & & $(11)$ & $(7)(11)$ & $(7)(11)$ \\
sor. & & $(24) 42.3 \%$ & $(24) 38.4 \%$ \\
\hline
\end{tabular}

\begin{tabular}{|llll|}
\hline \multicolumn{4}{|c|}{ Imágenes Colposcópicas } \\
\hline LESION & $\begin{array}{c}\text { Base y Punt. } \\
\text { Irregular }\end{array}$ & Vasos Atípicos & Erosión \\
\hline Cervicitis & $(24) 1.8 \%$ & $(24) 0.6 \%$ \\
Nic I & $(24) 15.1 \%$ & $(24) 0.7 \%$ \\
Nic II & $(24) 15.1 \%$ & $(24) 0.7 \%$ \\
Nic III & $(24) 59.8 \%$ & $(24) 16.4 \%$ \\
Microinvasivo & $(24) 69.2 \%$ & $(7) 31 \%,(8) 67-100 \%$ \\
Ca. Invasor & $(24) 12.8 \%$ & $(12) 76.9 \%$ & $(11)(19)(22)$ \\
& & $(24) 96.6 \%$ & $(21)$ \\
& & & $(2.5 \%$ \\
\hline
\end{tabular}

Para establecer en cuantos casos la citología fue mayor, igual o menor que la Anatomía Patológica, se discrimina en tablas diferentes todas las opciones diagnósticas de NIC.

También se discrimina en una tabla diferente, las imágenes colposcópicas frente a las diferentes opciones diagnósticas dada por Anatomía Patológica.

Para el análisis y en el caso de que la paciente tenga una o más citologías, una o más biopsias o resultados de Anatomía Patológica, se tomará la que presente lesión más severa.

Se desecharon del estudio, las colposcopias realizadas por personal inexperto.

Los resultados de citología y biopsia, así como la Anatomía Patológica están sujetos a los criterios que rigen en nuestro departamento de patología.

\section{Definición de términos}

- Citología positiva: Se considera citologías A3-A4 y A5.

- Citología negativa: A1 y A2 (con o sin atipias).

- CP (Colposcopia) Satisfactoria: cuando se observan los límites de la zona de transformación en toda su extensión, con evidencia de los bordes de la lesión.

- CP Insatisfactoria: No se observan los límites de la zona de transformación o límites de la lesión, pues aquella se introduce profundo en el canal.
- El resultado de las biopsias y piezas se hará de acuerdo con la clasificación internacional de NIC y Ca. de cervix.

- Lesión colposcópica benigna: incluye los parámetros de hallazgos colposcópicos normales (epitelio escamoso original, epitelio columnar normal, zona de transformación típica) o lesión inflamatoria, según los congresos de Graz (1975) y el congreso de colposcopia de 1981 .

- Lesión colposcópica anormal; comprende, lo enunciado en la nomenclatura de estos mismos congresos, como hallazgos colposcópicos anormales (Zonas de transformación atípica: mosaico, puntuación, epitelio blanco, keratosis, vasos atípicos) o sospechosa de Ca. invasivo (vasos atípicos, erosión o úlcera).

- Imágenes colposcópicas: las imágenes colposcópicas aquí descritas, se ciñen a la nomenclatura internacional extraída de los congresos antes mencionados.

- Zona de transformación típica: Ectropión en vía de curación por proliferación de epitelio pavimentoso que la rodea, por diferenciación pavimentosa de las células de reserva o por ambos procesos. Es una superficie irregular, con orificios glandulares abiertos y cerrados y quistes de Naboth, con una trama vascular regular.

- Zona de transformación atípica: Incluye los hallazgos de lesiones atípicas en la zona de transformación: Mosaico, puntuación, epitelio blanco, keratosis, y vasos atípicos.

(imágenes matrices de Hinselman).

\section{Resultados}

De acuerdo con los parámetros expuestos se revisaron 400 casos de las pacientes atendias en la clínica de oncología del Instituto Materno Infantil de Bogotá, con sus respectivas colposcopias y resultados de Anatomía Patológica.

En 16 pacientes no se realizó citología, siendo pacientes que ingresaron por el servicio de urgencias con un carcinoma de cervix invasivo y sangrante.

De los 400 casos el resultado final de patología se estableció de la siguiente manera:

$\begin{array}{lrr} & \text { No. } & \% \\ \text { Biopsia } & 315 & 79 \\ \text { Conos } & 48 & 12 \\ \text { Histerectomía } & 37 & 9 \\ \text { TOTAL } & 400 & 100\end{array}$

La discriminación de casos por lesiones definitivas se establece en la tabla No. 2.

Es de anotar que las lesiones benignas corresponden en su mayoría a Cervicitis aguda y crónica con 104 casos, que corresponden al $26 \%$ de toda la muestra. Las demás lesiones benignas fueron reportadas por los patólogos como:

- Atipias benignas

- Metaplasia escamosa.

- Hiperplasia de células endocervicales.

- Epitelio normal

- Lesión por condiloma.

\section{CITOLOGIA:}

Comparación de la citología frente al resultado final de anatomía patológica: Los resultados se exponen en el Cuadro No. 1.

Las pruebas de significancia para la citología frente al resultado final de patología, nos demuestran: 
No. $\%$

Sensibilidad

$247 / 266$

93

Especificidad

$50 / 118$

42

Valor predictivo (+ ) $247 / 315 \quad 78$

$\begin{array}{lll}\text { Valor predictivo ( }- \text { ) } & 50 / 69 & 72\end{array}$

falsos positivos $\quad 68 / 118 \quad 58$

falsos negativos $\quad 19 / 266 \quad 7$

$\left(\mathrm{X}^{2}=68,8 \ldots \ldots \ldots . \mathrm{p}<0.001\right)$

Tabla No. 2:

FRECUENCIA DE CASOS

POR LESION DEFINITIVA:

\begin{tabular}{|lrr|}
\hline & No. & \% \\
\hline Lesión benigna & 118 & 29.5 \\
NIC I & 44 & 11.0 \\
NIC II & 38 & 9.5 \\
NIC III & 149 & 37.0 \\
Microinvasivo & 3 & 1.0 \\
Carcinoma invasivo & 48 & 12.0 \\
\hline TOTAL & 400 & 100.0 \\
\hline
\end{tabular}

Cuadro No. 1:

COMPARACION DE LA CITOLOGIA FRENTE AL RESULTADO FINAL DE PATOLOGIA:

\begin{tabular}{clrrrrr}
\hline \multirow{2}{*}{ Citología } & \multicolumn{5}{c}{ Patología } \\
\cline { 2 - 7 } & \multicolumn{2}{c}{$(+)$} & \multicolumn{2}{c}{$(=)$} & \multicolumn{2}{c}{ Total } \\
\cline { 2 - 7 } & No. & $\%$ & No. & $\%$ & No. & $\%$ \\
\hline$(+)$ & 247 & 64 & 68 & 18 & 315 & 82 \\
$(=)$ & 19 & 5 & 50 & 13 & 69 & 18 \\
Total & 266 & 69 & 118 & 31 & 384 & 100 \\
\hline
\end{tabular}

Comparando el resultado final de citología y patología, en cuanto si la lesión fue mayor o menor, se describen los resultados en el Cuadro No. 2.

De esta se extrae:

$\begin{array}{lrr} & \text { No. } & \% \\ \text { Citología = Patología } & 182 & 47 \\ \text { Citologia }>\text { Patología } & 77 & 20 \\ \text { Citología < Patología } & 126 & 33\end{array}$

La frecuencia de citología negativa según las diferentes patologías, fue:

$\begin{array}{llr} & \text { No. } & \% \\ \text { NIC I } & 6 / 44 & 14 \\ \text { NIC II } & 4 / 38 & 11 \\ \text { NIC III } & 6 / 149 & 4 \\ \text { Microinvasivo } & - & 0 \\ \text { Ca. Invasivo } & 3 / 32 & 9\end{array}$

Correlación entre citología A2 con atipias severas y resultado final de patología: La correlación entre los casos

con Citología A2 con Atípias severas y las diferentes lesiones se establece en la tabla No. 3 .

Correlación entre citología y carcinoma invasivo: La relación entre citología A5 y patología final confirmatoria de Ca. Invasivo se describe en el Cuadro No. 3.

Las pruebas de sensibilidad para la citología A5 para diagnóstico de $\mathrm{Ca}$. Invasivo, son:

Sensibilidad
Especificidad
Valor pred. (+)
Valor pred. (- )
Falsos ( + )
Falsos ( - )

$\begin{array}{cr}\text { No. } & \% \\ 9 / 16 & 56 \\ 345 / 368 & 94 \\ 9 / 32 & 28 \\ 345 / 352 & 98 \\ 23 / 368 & 6 \\ 7 / 16 & 44\end{array}$

$$
\left(\mathrm{X}^{2}=50.2 \ldots . \mathrm{P}<0.001\right)
$$

La correlación entre citología A4-A5 y patología final confirmatoria de Ca.Invasivo se describe en el Cuadro No.4.

Las pruebas de sensibilidad para citología A4-A5 para diagnóstico de Ca. Invasivo, son:

$$
\begin{aligned}
& \text { Sensibilidad } \\
& \text { Especificidad } \\
& \text { Valor pred. (+) } \\
& \text { Valor pred. (-) } \\
& \text { Falsos (+) } \\
& \text { Falsos (-) }
\end{aligned}
$$

$\begin{array}{rr}\text { No. } & \% \\ 22 / 92 & 24 \\ 282 / 352 & 80 \\ 22 / 32 & 69 \\ 282 / 352 & 80 \\ 10 / 292 & 3 \\ 70 / 92 & 76\end{array}$

$\left(\mathrm{X}^{2}=35.15 \ldots \ldots \ldots . \mathrm{P}<0.001\right)$

Cuadro No. 2:

COMPARACION DEL RESULTADO

DE CITOLOGIA Y PATOLOGIA

\begin{tabular}{lccccccc}
\hline & \multicolumn{9}{c}{ Citología } \\
\cline { 2 - 8 } Patología & A1 & A2 & A3 & A4 & A5 & \multicolumn{3}{c}{ Total } \\
\cline { 3 - 8 } & & & & & & No. & $\%$ \\
\hline Lesión Benigna & 2 & 48 & 62 & 5 & 1 & 118 & 31 \\
Nic I & - & 6 & 37 & 1 & - & 44 & 11 \\
Nic II & - & 4 & 32 & 2 & - & 38 & 10 \\
Nic III & 1 & 5 & 84 & 53 & 6 & 149 & 39 \\
Microinvasivo & - & - & 1 & 2 & - & 3 & 1 \\
Ca. Invasivo & - & 3 & 7 & 13 & 9 & 32 & 8 \\
\hline Total (\%) & $3(1)$ & $66(17)$ & 223 & $76(20)$ & $16(4)$ & 384 & $(100)$ \\
& & & $(58)$ & & & & \\
\hline
\end{tabular}


Tabla No. 3

CORRELACION ENTRE CITOLOGIA A2 CON ATIPIAS SEVERAS Y LESION FINAL POR PATOLOGIA

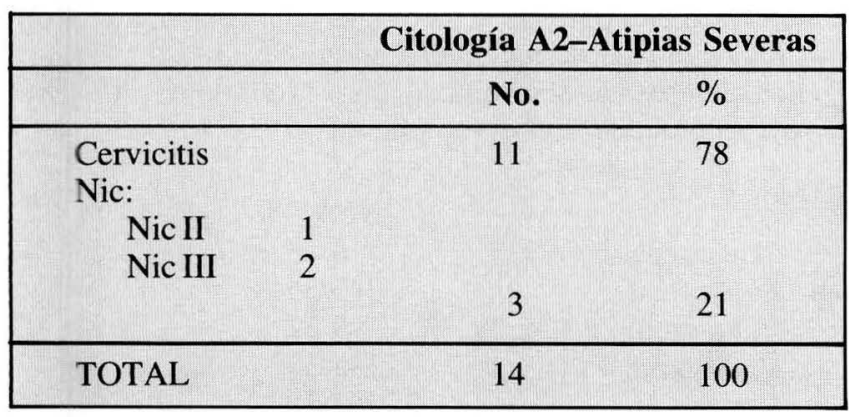

Cuadro No. 3

CORRELACION ENTRE CITOLOGIA A5 Y CARCINOMA INVASIVO

\begin{tabular}{rlrrrrr}
\hline & \multicolumn{5}{c}{ Citología A5 } \\
\cline { 2 - 7 } Ca. Invasivo & \multicolumn{2}{c}{$(+)$} & \multicolumn{2}{c}{$(-)$} & \multicolumn{2}{c}{ Total } \\
\cline { 2 - 7 } & No. & $\%$ & No. & $\%$ & No. & $\%$ \\
\hline$(+)$ & 9 & 2 & 23 & 6 & 32 & 8 \\
$(-)$ & 7 & 2 & 245 & 90 & 352 & 92 \\
\hline Total & 16 & 4 & 368 & 96 & 384 & 100 \\
\hline
\end{tabular}

CORRELACION ENTRE CITOLOGIA A4-A5 Y CARCINOMA INVASIVO

\begin{tabular}{ccccccr}
\hline & \multicolumn{6}{c}{ Citología A5 } \\
\cline { 2 - 7 } Ca. Invasivo & \multicolumn{2}{c}{$(+\varsigma$} & \multicolumn{2}{c}{$(-)$} & \multicolumn{2}{c}{ Total } \\
\cline { 2 - 7 } & No. & $\%$ & No. & $\%$ & No. & $\%$ \\
\hline$(+)$ & 22 & 6 & 10 & 3 & 32 & 9 \\
$(-)$ & 70 & 18 & 282 & 73 & 352 & 91 \\
\hline Total & 92 & 24 & 292 & 76 & 384 & 100 \\
\hline
\end{tabular}

Correlación entre citología y colposcopia: Para establecer las pruebas de sensibilidad de la colposcopia frente a la citología, se establece la correlación entre lesión colposcópicamente anormal y lesión colposcópica benigna en relación con la positividad de la citología. Cuadro No. 5.

Las pruebas de sensibilidad de la colposcopia frente a la citología, se refieren:

$\begin{array}{lll} & \text { No. } & \% \\ \text { Sensibilidad } & 266 / 301 & 88 \\ \text { Especificidad } & 32 / 83 & 39 \\ \text { Valor pred. (+) } & 266 / 317) & 84 \\ \text { Valor pred. (-) } & 32 / 67 & 48 \\ \text { Falsos (+) } & 51 / 83 & 61 \\ \text { Falsos (-) } & 35 / 301 & 12 \\ & \left(\mathrm{X}^{2}=281 \ldots \ldots . \mathrm{p}<0.001\right)\end{array}$

\section{BIOPSIAS:}

Correlación entre biopsia y resultado final de patología: La correlación entre la positividad de la biopsia y la pieza final de anatomía patológica, se refiere en el Cuadro No. 6 .

Las pruebas de significancia para esta prueba, son:

$\begin{array}{lll} & \text { No. } & \% \\ \text { Sensibilidad } & 79 / 87 & 91 \\ \text { Especificidad } & 6 / 16 & 38 \\ \text { Valor Pred. (+) } & 79 / 89 & 89 \\ \text { Valor Pred. (-) } & 6 / 14 & 43 \\ \text { Falsos (+) } & 10 / 16 & 63 \\ \text { Falsos (-) } & 8 / 87 & 9 \\ & & \\ & \left(\mathrm{X}^{2}=9.2 \ldots \ldots . \mathrm{p}<0.01\right)\end{array}$

Correlación entre grado de lesión encontrado en la biopsia y en la pieza final de anatomía patológica: La correlación entre las lesiones encontradas en la biopsia frente a la pieza final, se exponen en el Cuadro No. 7.

En relación con el grado de lesión se encontró:

$$
\begin{aligned}
& \text { No. } \quad \% \\
& \text { Biopsia }=\text { Patología } 72 \\
& \text { Biopsia<Patología } 16 \\
& \text { Biopsia<Patología } 15 \quad 15 \\
& \begin{array}{lll}
\text { Total } & 103 & 100
\end{array} \\
& \text { Cuadro No. } 5 \\
& \text { CORRELACION ENTRE POSITIVIDAD } \\
& \text { DE CITOLOGIA FRENTE A POSITIVIDAD } \\
& \text { DE LA COLPOSCOPIA }
\end{aligned}
$$

\begin{tabular}{cllllll}
\hline & \multicolumn{5}{c}{ Colposcopia } \\
\cline { 2 - 7 } Citología & Les. Anormal & Les. Benigna & \multicolumn{2}{c}{ Total } \\
\cline { 2 - 7 } & No. & $\%$ & No. & $\%$ & No. & $\%$ \\
\hline$(+)$ & 256 & 69 & 51 & 13 & 317 & 82 \\
$(-)$ & 35 & 9 & 32 & 8 & 67 & 17 \\
\hline Total & 301 & 78 & 83 & 21 & 384 & 99 \\
\hline
\end{tabular}

$$
\text { Cuadro No. } 6
$$

CORRELACION ENTRE POSITIVIDAD

DE LA BIOPSIA Y DE LA PIEZA FINAL

DE ANATOMIA PATOLOGICA: (AP):

\begin{tabular}{crrrrrrr}
\hline & \multicolumn{6}{c}{ AP } \\
\cline { 2 - 7 } Biopsia & \multicolumn{2}{c}{$(+)$} & \multicolumn{2}{c}{$(-)$} & \multicolumn{2}{c}{ Total } \\
\cline { 2 - 7 } & No. & $\%$ & No. & $\%$ & No. & $\%$ \\
\hline & 79 & 76 & 10 & 10 & 89 & 86 \\
$(+)$ & 8 & 8 & 6 & 6 & 14 & 14 \\
\hline Total & 87 & 84 & 16 & 16 & 103 & 100 \\
\hline
\end{tabular}




\begin{abstract}
Cuadro No. 7
CORRELACION ENTRE TIPO DE LESION

ENCONTRADA EN LA BIOPSIA Y PIEZA FINAL DE ANATOMIA PATOLOGICA
\end{abstract}

\begin{tabular}{|c|c|c|c|c|c|c|c|c|}
\hline \multirow{3}{*}{ Biopsia } & \multicolumn{8}{|c|}{ Pieza final } \\
\hline & \multirow{2}{*}{$(-)$} & \multirow{2}{*}{$\begin{array}{c}\text { Nic } \\
\text { I }\end{array}$} & \multirow{2}{*}{$\begin{array}{c}\text { Nic } \\
\text { II }\end{array}$} & \multirow{2}{*}{$\begin{array}{l}\text { Nic } \\
\text { III }\end{array}$} & \multirow{2}{*}{$\begin{array}{l}\text { Micro- } \\
\text { invasi. }\end{array}$} & \multirow{2}{*}{$\begin{array}{l}\text { Inva- } \\
\text { sivo }\end{array}$} & \multicolumn{2}{|c|}{ Total } \\
\hline & & & & & & & No. & $\%$ \\
\hline$(-)$ & 6 & 2 & 4 & 2 & - & - & 14 & 14 \\
\hline $\mathrm{Nic} I$ & 2 & 1 & 2 & 1 & - & - & 6 & 6 \\
\hline Nic II & 2 & - & 1 & 1 & - & - & 4 & 4 \\
\hline Nic III & 6 & 1 & 5 & 61 & 2 & 1 & 76 & 73 \\
\hline Microinvasivo & - & - & - & - & 1 & - & 1 & 1 \\
\hline Invasivo & - & - & - & - & - & 2 & 2 & 2 \\
\hline Total $(\%)$ & $\begin{array}{l}16 \\
(15)\end{array}$ & $4(4)$ & $\begin{array}{c}12 \\
(12)\end{array}$ & $65(6$ & $3) 3(3)$ & $3(3)$ & 103 & 100 \\
\hline
\end{tabular}

\section{COLPOSCOPIA:}

Frecuencia de colposcopia insatisfactoria: De los 400 casos, encontramos:

Colposcopia satisfactoria: 264 casos, $66 \%$

Colposcopia insatisfactoria: 136 , casos para el $34 \%$.

La distribución de colposcopia según la edad se encuentra en el Cuadro No. 8. y en la Gráfica No. 1.

De este se extrae:

$$
\begin{aligned}
& \text { Pacientes de más de } 40 \text { años: } \\
& \text { CP Satisfactorio } 79 \\
& \text { CP Insatisfactoria } 86 \\
& \text { No. } \quad \% \\
& \begin{array}{l}
79 \quad 48 \\
96
\end{array} \\
& 145 \quad 100
\end{aligned}
$$

Las pacientes mayores de 40 años corresponden al $41 \%$ de toda la muestra.

- En Pacientes menores de 40 años:

$$
\begin{array}{lll} 
& \text { No. } & \% \\
\text { CP Satisfactoria } & 185 & 79 \\
\text { CP Insatisfactoria } & 50 & 21
\end{array}
$$

$$
\begin{array}{lll}
\text { Total } & 235 & 100
\end{array}
$$

Relación de colposcopia con lesión positiva a nivel del canal endocervical: La relación entre colposcopia insatisfactoria y lesión en el canal se expresa en el Cuadro No. 9.

Las patologías para determinar compromiso del canal endocervical fueron: Biopsia: 32 casos

$$
\text { Conos: } 7 \text { casos }
$$

Correlación entre lesión encontrada por colposcopia y biopsia o pieza quirúrgica, para lesión final: Esta relación se establece en el Cuadro No. 10. De este se extraen las pruebas de sensibilidad para la colposcopia así:

$\begin{array}{lll} & \text { No. } & \% \\ \text { Sensibilidad } & 253 / 283 & 89 \\ \text { Especificidad } & 75 / 117 & 64 \\ \text { Valor pred. (+) } & 253 / 295 & 86 \\ \text { Valor pred. (-) } & 75 / 105 & 71 \\ \text { Falsos (+) } & 42 / 117 & 36 \\ \text { Falsos (-) } & 30 / 283 & 11\end{array}$

$$
\left(X^{2}=236.5 \ldots \ldots . p<0.001\right)
$$

Cuadro No. 8

DISTRIBUCION DE COLPOSCOPIA

SEGUN LAS DIFERENTES EDADES

\begin{tabular}{lllllllll}
\hline & \multicolumn{8}{c}{ Edad } \\
\cline { 2 - 9 } Colposcopia & \multirow{2}{*}{$\mathbf{2 0}$} & $\mathbf{2 1 - 3 0}$ & $\mathbf{3 1 - 4 0}$ & $\mathbf{4 1 - 5 0}$ & $\mathbf{5 1 - 6 0}$ & $\mathbf{6 1 -}+$ & \multicolumn{2}{c}{ Total } \\
\cline { 3 - 9 } & & & & & & & No. & $\%$ \\
\hline Satisfactor. & 6 & 79 & 100 & 42 & 17 & 20 & 264 & 66 \\
Insatisfac. & - & 14 & 36 & 35 & 40 & 11 & 136 & 34 \\
\hline Total & 6 & 93 & 136 & 77 & 57 & 34 & 400 & 100 \\
$(\%)$ & $(1.5)$ & $(23)$ & $(34)$ & $(19)$ & $(14)$ & $(8.5)$ & \\
\hline
\end{tabular}

Gráfica No. 1

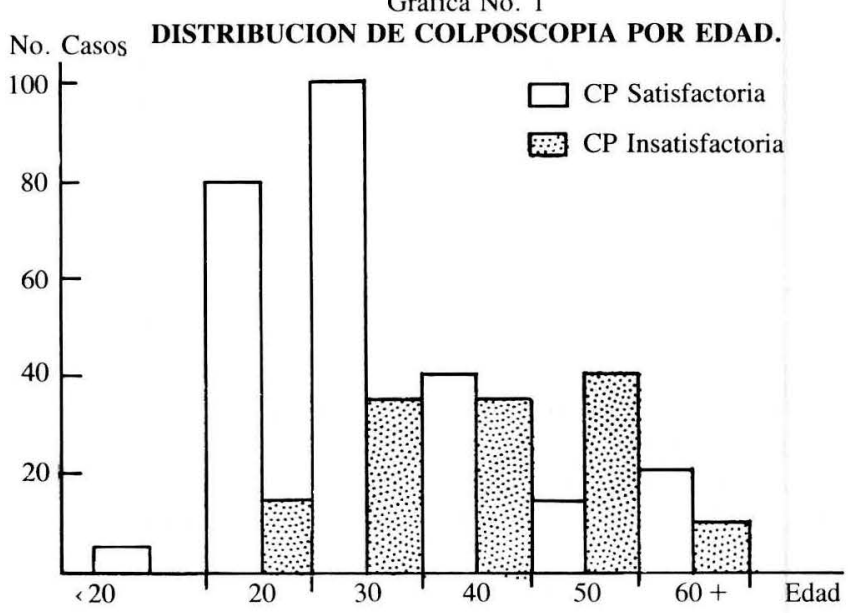

Cuadro No. 9

CORRELACION ENTRE COLPOSCOPIA

INSATISFACTORIA Y LESION EN EL CANAL

\begin{tabular}{lll}
\hline \multirow{2}{*}{ Colposcopia } & \multicolumn{2}{c}{ Lesión Endocervical positiva } \\
\cline { 2 - 3 } & No. & $\%$ \\
\hline Satisfactoria & 13 & 33 \\
Insatisfactoria & 26 & 67 \\
\hline Total & 39 & 100 \\
\hline
\end{tabular}

Cuadro No. 10

CORRELACION ENTRE LESION ENCONTRADA POR COLPOSCOPIA Y RESULTADO FINAL DE PATOLOGIA:

\begin{tabular}{lllllll}
\hline & \multicolumn{5}{c}{ Patología } \\
\cline { 2 - 7 } Colposcopia & \multicolumn{2}{c}{$(+)$} & \multicolumn{2}{c}{$(-)$} & \multicolumn{2}{c}{ Total } \\
\cline { 2 - 7 } & No. & $\%$ & No. & $\%$ & No. & $\%$ \\
\hline Les. anormal & 253 & 63 & 42 & 10.5 & 295 & 73.5 \\
Les. beningna 30 & 7.5 & 75 & 19 & 105 & 26.5 \\
\hline Total ( \% ) & 283 & 70.5 & 117 & 29.5 & 400 & 100 \\
\hline
\end{tabular}

4. CONDILOMA:

Correlación entre citología positiva para coilocitos y patología final positiva para condiloma: Esta correlación se 
establece en el Cuadro No. 11. los resultados de patología positivos para displasia coilocítica se obtuvieron así:

$\begin{array}{llr}- \text { Biopsia } & = & 56 \text { casos } \\ \text {-Cono } & = & 12 \text { casos } \\ \text {-Histerectomía } & = & 8 \text { casos } \\ & & 76 \text { casos }\end{array}$

Es de anotar que la citología solo fue positiva para 13 casos en las que se reporta coilocitos y de éstas sólo 9 fueron positivas para displasia coilocítica en la biopsia o pieza final.

Las pruebas de sensibilidad de la citología para condiloma, se establecen así:

$\begin{array}{lll} & \text { No. } & \% \\ \text { Sensibilidad } & 9 / 76 & 12 \\ \text { Especificidad } & 304 / 308 & 99 \\ \text { Valor predic. }(+) & 9 / 13 & 69 \\ \text { Valor predic. }(-) & 304 / 371 & 82 \\ \text { Falsos (+) } & 4 / 308 & 1 \\ \text { Falsos (-) } & 67 / 76 & 88 \\ & & \\ & \left(\mathrm{X}^{2}=20.6 \ldots \ldots \ldots . \mathrm{p}<0.001\right)\end{array}$

Correlación entre imagen colposcópica positiva para condiloma y patología final confirmatoria de condiloma: Esta correlación se expone en el Cuadro No. 12. Las pruebas de significancia para la colposcopia en condiloma frente a la patología final, son:

$\begin{array}{lll} & \text { No. } & \% \\ \text { Sensibilidad } & 48 / 76 & 63 \\ \text { Especificidad } & 276 / 324 & 85 \\ \text { Valor Pred. (+) } & 48 / 96 & 50 \\ \text { Valor Pred. (-) } & 276 / 304 & 91 \\ \text { Falsos (+) } & 48 / 324 & 15 \\ \text { Falsos (-) } & 28 / 76 & 37 \\ & & \\ & \left(\mathrm{X}^{2}=78.3 \ldots \ldots \ldots \mathrm{p}<0.001\right)\end{array}$

Encontramos 96 colposcopias sugestivas de condiloma, con dos imágenes base de dicha patología:

- El seudomosaico

- Area papilar o papilífera

Las diferentes imágenes referidas por los colposcopistas corresponden a:

- Area papilar gruesa

- Proyecciones papilíferas

- Micropapulas

- Zona papilífera

- Aspecto papilífero con vasos circulares

- Area papilar blanca con vaso doble

- Area papilar con vaso central en asa doble

- Papilas con vasos puntiformes

- Epitelio acetoblanco con área papilífera brillante

- Area papilar con punteado rojo
- Neovascularización en área papilar

- Vesículas con vasos en capullo

- Vesículas con vasos en capullo

- Imagen de gotas de cera

- Hiperqueratosis más seudomosaico

- Vesículas hipertróficas

- Microvesículas

Anotamos que las pacientes que presentan lesión positiva por anatomía patológica, en 70 casos (92\%), la colposcopia reveló Leucoplasia.

\section{COMPROMISO GLANDULAR:}

En la biopsia o pieza final, se encontró en 56 casos reporte de compromiso glandular, distribuidos los casos así:

Biopsia

No.

Pieza final 26

8

Biopsia con compromiso glandular y pieza final sin compromiso

Biopsia con compromiso glandular y pieza final con compromiso

De los 22 casos, en los que se practicó cono o histerectomía luego de la biopsia con compromiso glandular, (22 casos), en ninguno se comprobó invasión en la pieza final.

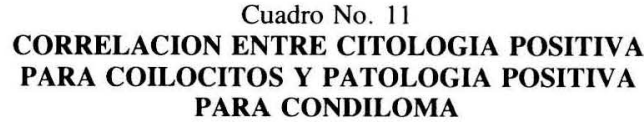

\begin{tabular}{lllllll}
\hline \multirow{2}{*}{ Citología } & \multicolumn{6}{c}{ Patología } \\
\cline { 2 - 7 } & \multicolumn{2}{c}{$(=)$} & \multicolumn{2}{c}{$(-)$} & \multicolumn{2}{c}{ Total } \\
\cline { 2 - 7 } & No. & $\%$ & No. & $\%$ & No. & $\%$ \\
\hline$(+)$ & 9 & 2 & 4 & 1 & 13 & 3 \\
$(-)$ & 67 & 18 & 304 & 79 & 371 & 97 \\
\hline Total & 76 & 20 & 308 & 80 & 354 & 100 \\
\hline
\end{tabular}

\section{CORRELACION DE IMAGENES COLPOSCOPICAS}

\section{$Y$ LESION PATOLOGICA}

La frecuencia de imágenes colposcópicas según la patología se discrimina en el Cuadro No. 13.

La frecuencia (porcentaje) de imágenes más sobresalientes por lesión se dan en la tabla No. 4.

Imágenes colposcópicas en el Nic: En el Cuadro No. 14, se correlaciona la positividad en la patología para Nic y la leucoplasia.

Las pruebas de significancia para la leucoplasia en relación con el NIC son:

\begin{tabular}{|c|c|c|c|c|}
\hline & No. & & & \\
\hline Sensibilidad & $203 / 23188$ & Valor pred. $(-)$ & $94 / 122$ & 77 \\
\hline Especificidad & $94 / 118 \quad 80$ & V.Falsos (+) & $24 / 118$ & 20 \\
\hline Valor pred. $(+)$ & $203 / 22789$ & Falsos $(-)$ & $28 / 231$ & 12 \\
\hline
\end{tabular}


- En el Cuadro No. 15, se correlaciona la positividad en la patología para Nic y el punteado.

Las pruebas de sensibilidad para el punteado en relación con el Nic son:

$\begin{array}{lll} & \text { No. } & \% \\ \text { Sensibilidad } & 124 / 231 & 54 \\ \text { Especificidad } & 94 / 118 & 80 \\ \text { Valor pred. ( }) & 124 / 148 & 84 \\ \text { Valor pred. (-) } & 94 / 201 & 47 \\ \text { Falsos (+) } & 24 / 118 & 20 \\ \text { Falsos (-) } & 107 / 231 & 46 \\ & & \\ & \left(\mathrm{X}^{2}=35.5 \ldots \ldots \ldots \mathrm{p}<0.001\right)\end{array}$

- En el Cuadro No. 16 se correlaciona la positividad en la patología para Nic y el mosaico.

Las pruebas de sensibilidad para esta imagen frente al Nic son:

$\begin{array}{lll} & \text { No. } & \% \\ \text { Sensibilidad } & 54 / 231 & 23 \\ \text { Especificidad } & 115 / 118 & 97 \\ \text { Valor pred. (+) } & 54 / 57 & 94 \\ \text { Valor pred. (-) } & 115 / 292 & 39 \\ \text { Falsos (+) } & 3 / 118 & 3 \\ \text { Falsos (-) } & 177 / 231 & 77 \\ & & \\ & \left(\mathrm{X}^{2}=24.8 \ldots \ldots . \mathrm{p}<0.001\right) .\end{array}$

Cuadro No. 12

CORRELACION ENTRE IMAGEN COLPOSCOPICA POSITIVA PARA CONDILOMA Y LESION DE PATOLOGIA CONFIRMATORIA DE DISPLASIA COILOCITICA

\begin{tabular}{ccccccc}
\hline \multirow{2}{*}{$\begin{array}{c}\text { Colposcopia: } \\
\text { Imagen de } \\
\text { Condiloma }\end{array}$} & \multicolumn{4}{c}{$\begin{array}{c}\text { Patología: Displasia coilocítica } \\
\text { (Condiloma) }\end{array}$} \\
\cline { 2 - 7 } & \multicolumn{1}{c}{$(+)$} & \multicolumn{2}{c}{$(-)$} & \multicolumn{4}{c}{ Total } \\
\cline { 2 - 7 } & No. & $\%$ & No. & $\%$ & No. & $\%$ \\
\hline$(+)$ & 48 & 12 & 48 & 12 & 96 & 24 \\
$(-)$ & 28 & 7 & 276 & 69 & 304 & 76 \\
\hline Total & 76 & 19 & 324 & 81 & 400 & 100 \\
\hline
\end{tabular}

\begin{tabular}{|c|c|c|c|c|c|c|c|c|c|}
\hline \multirow{3}{*}{$\mathbf{C P}$} & \multicolumn{9}{|c|}{ Patología } \\
\hline & \multirow{2}{*}{$\begin{array}{l}\text { Les. } \\
\text { Benig- } \\
\text { na }\end{array}$} & \multirow{2}{*}{$\begin{array}{l}\text { Cer- } \\
\text { vici } \\
\text { tis }\end{array}$} & \multirow{2}{*}{$\underset{\text { I }}{\text { Nic }}$} & \multirow[b]{2}{*}{ II } & \multirow[b]{2}{*}{ III } & \multirow{2}{*}{$\begin{array}{l}\text { Micro- } \\
\text { invasi. }\end{array}$} & \multirow{2}{*}{$\begin{array}{c}\text { Ca. } \\
\text { Inva- } \\
\text { sor }\end{array}$} & \multicolumn{2}{|c|}{ Total } \\
\hline & & & & & & & & No. & $\%$ \\
\hline Endopía & 4 & 13 & 6 & 2 & 18 & - & 2 & 45 & 11 \\
\hline Ectopía & 5 & 44 & 18 & 14 & 45 & - & 2 & 128 & 32 \\
\hline ZTT & 3 & 78 & 30 & 24 & 87 & 2 & 8 & 232 & 58 \\
\hline Colpitis & 3 & 38 & 18 & 14 & 48 & 1 & 8 & 130 & 33 \\
\hline \multicolumn{10}{|l|}{ Epitelio } \\
\hline Acetoblanco & 5 & 47 & & & & & & 52 & 13 \\
\hline Leucoplasia & 1 & 23 & 30 & 31 & 141 & 2 & 34 & 263 & 66 \\
\hline Punteado & 1 & 23 & 10 & 14 & 100 & 1 & 24 & 173 & 43 \\
\hline Mosaico & - & 3 & 5 & 5 & 44 & 1 & 8 & 66 & 17 \\
\hline Vasos III & 2 & 31 & 14 & 10 & 35 & - & - & 92 & 23 \\
\hline Vasos IV & - & - & 6 & 6 & 36 & 2 & 35 & 85 & 21 \\
\hline Vasos V & - & - & - & 3 & 5 & - & 24 & 32 & 8 \\
\hline Ulcera & - & - & - & - & - & - & 16 & 16 & 4 \\
\hline \multicolumn{10}{|l|}{ Proliferac. } \\
\hline Carcinomatosa & - & - & - & - & 1 & 1 & 32 & 34 & 9 \\
\hline $\begin{array}{l}\text { Total } \\
(\%)\end{array}$ & $\begin{array}{l}14 \\
(3)\end{array}$ & $\begin{array}{l}104 \\
(26)\end{array}$ & $\begin{array}{c}44 \\
(11)\end{array}$ & $\begin{array}{l}38 \\
(10)\end{array}$ & $\begin{array}{l}149 \\
(37)\end{array}$ & $\begin{array}{l}3 \\
(1)\end{array}$ & $\begin{array}{l}48 \\
(12)\end{array}$ & 400 & \\
\hline
\end{tabular}


Tabla No. 4

ORDEN DE FRECUENCIA DE LESIONES COLPOSCOPICAS SEGUN TIPO DE LESION

Cervicitis

Epitelio acetoblanco

$\%$

Zona transformación típica

Vasos III

Colpitis

punteado

NIC I:

Leucoplasia

Vasos III

Punteado

Vasos IV

Mosaico

NIC II:

Leucoplasia

Punteado

Vasos III

Vasos IV

Mosaico

NIC III:

Leucoplasia

Punteado

Mosaico

Vasos III

Vasos IV

Vasos V

Microinvasivo:

Leucoplasia

Vasos IV

Punteado

Mosaico

Prolifer. Carcinomatosa

Ca. Invasivo:

Vasos IV

Leucoplasia

Prolifer. Carcinomatosa

Punteado

Vasos V

Ulcera

Mosaico

Cuadro No. 15

CORRELACION ENTRE PUNTEADO

Y TODOS LOS CASOS DE NIC

\section{Patología Nic}

\begin{tabular}{ccccccc}
\multirow{2}{*}{ Punteado } & \multicolumn{2}{c}{$(+)$} & \multicolumn{2}{c}{$(-)$} & \multicolumn{2}{c}{ Total } \\
\cline { 2 - 7 } & No. & $\%$ & No. & $\%$ & No. & $\%$ \\
\hline$(+)$ & 124 & 36 & 24 & 7 & 148 & 43 \\
$(-)$ & 107 & 30 & 94 & 27 & 201 & 57 \\
\hline Total & 231 & 66 & 118 & 34 & 349 & 100 \\
\hline
\end{tabular}

Cuadro No. 16

CORRELACION ENTRE MOSAICO Y TODOS LOS CASOS DE NIC

\begin{tabular}{ccccccc}
\hline & \multicolumn{6}{c}{ Patología Nic } \\
\cline { 2 - 7 } Mosaico & \multicolumn{2}{c}{$(+)$} & \multicolumn{2}{c}{$(-)$} & \multicolumn{2}{c}{ Total } \\
\cline { 2 - 7 } & No. & $\%$ & No. & $\%$ & No. & $\%$ \\
\hline$(+)$ & 54 & 15 & 3 & 1 & 57 & 16 \\
$(-)$ & 177 & 51 & 115 & 33 & 292 & 84 \\
\hline Total & 231 & 66 & 118 & 34 & 349 & 100 \\
\hline
\end{tabular}

Imágenes colposcópicas en el carcinoma invasivo: - La correlación entre vasos IV y diagnóstico de $\mathrm{Ca}$. invasivo por patología se establece en el Cuadro No. 17.

Las pruebas de significancia para esta imagen frente al Ca. invasivo nos refieren: No. $\quad \%$

$\begin{array}{lll}\text { Sensibilidad } & 35 / 48 & 73 \\ \text { Especificidad } & 302 / 352 & 86 \\ \text { Valor pred. ( }+) & 35 / 85 & 41 \\ \text { Valor pred. (-) } & 302 / 315 & 96 \\ \text { Falsos (+) } & 50 / 352 & 14 \\ \text { Falsos (-) } & 13 / 48 & 27 \\ & \left(\mathrm{X}^{2}=87 \ldots \ldots \ldots . . \mathrm{P}<0.001\right)\end{array}$

La correlación entre vasos $\mathrm{V}$ y diagnóstico de $\mathrm{Ca}$. Invasor por patologías se establece en el Cuadro No. 18.

Las pruebas de significancia para esta imagen frente al Ca. invasor nos refieren: No. $\%$

$\begin{array}{lll}\text { Sensibilidad } & 24 / 48 & 50 \\ \text { Especificidad } & 344 / 352 & 98 \\ \text { Valor pred. (+) } & 24 / 32 & 75 \\ \text { Valor pred. (-) } & 344 / 368 & 93 \\ \text { Falsos (+) } & 8 / 352 & 2 \\ \text { Falsos (-) } & 24 / 48 & 50\end{array}$

$$
\left(X^{2}=130 \ldots \ldots \ldots \ldots . p<0.001\right)
$$

La correlación entre la imagen de proliferación carcinomatosa y Ca. invasivo, se establece en el Cuadro No. 19. Las pruebas de significancia para esta imagen frente a la Ca. invasivo son:

Sensibilidad

Especificidad

No.

$31 / 48$

$350 / 352$

Valor pred. $(+) \quad 31 / 34$

Valor pred.(-) 350/366

Falsos (+)

$2 / 352$

Falsos (-)

$16 / 48$

$\%$

65

99

91

96

1

33

$\left(\mathrm{X}^{2}=222.5 \ldots \ldots \ldots \ldots \mathrm{p}<0.001\right)$

Cuadro No. 17

CORRELACION ENTRE VASOS IV Y CARCINOMA INVASOR

Patología de Ca. Invasivo

\begin{tabular}{cllllll}
\multirow{2}{*}{ VASOS I I } & \multicolumn{2}{c}{$(+)$} & \multicolumn{2}{c}{$(-)$} & \multicolumn{2}{c}{ Total } \\
\cline { 2 - 7 } & No. & $\%$ & No. & $\%$ & No. & $\%$ \\
\hline$(+)$ & 35 & 9 & 50 & 13 & 85 & 22 \\
$(-)$ & 13 & 3 & 302 & 75 & 315 & 78 \\
\hline Total & 48 & 12 & 352 & 89 & 400 & 99 \\
\hline
\end{tabular}


Cuadro No. 18

CORRELACION ENTRE VASOS $\mathrm{V}$

Y CARCINOMA INVASOR

\begin{tabular}{llrrrrr}
\hline & \multicolumn{4}{c}{ Patología de Ca. Invasivo } \\
\cline { 2 - 7 } Vasos V & \multicolumn{2}{c}{$(+)$} & \multicolumn{2}{c}{$(-)$} & \multicolumn{2}{c}{ Total } \\
\cline { 2 - 7 } & No. & $\%$ & No. & \% & No. & $\%$ \\
\hline$(+)$ & 24 & 6 & 8 & 2 & 32 & 8 \\
$(-)$ & 24 & 6 & 344 & 86 & 368 & 92 \\
\hline Total & 48 & 12 & 352 & 88 & 400 & 100 \\
\hline
\end{tabular}

Cuadro No. 19

CORRELACION ENTRE PROLIFERACION

CARCINOMATOSA Y DIAGNOSTICO DE CA INVASOR

\begin{tabular}{ccccccc}
\hline & \multicolumn{5}{c}{ Patología de Ca. Invasivo } \\
\cline { 2 - 7 } Proliferac. \\
\cline { 2 - 7 } Carcinomat & \multicolumn{2}{c}{$(+)$} & \multicolumn{2}{c}{$(-)$} & \multicolumn{2}{c}{ Total } \\
\cline { 2 - 7 } & No. & $\%$ & No. & $\%$ & No. & $\%$ \\
\hline$(+)$ & 32 & 8 & 2 & 1 & 34 & 9 \\
$(-)$ & 16 & 4 & 350 & 87 & 366 & 91 \\
\hline Total & 48 & 12 & 352 & 88 & 400 & 100 \\
\hline
\end{tabular}

\section{Discusión}

La frecuencia de patologías definitivas, corresponde a una frecuencia mayor de NIC III con el $37 \%$, justificada dicha frecuencia por el Instituto un centro de referencia de Neopasia intraepitelial.

Encontramos una frecuencia alta de Ca. invasivo (12\%) y una frecuencia de microinvasión acorde con la literatura $(1 \%)$.

La citología en el estudio demuestra una sensibilidad diagnóstica del 93\%, semejante a lo refererido por otros estudios (92.6\% (29)).

La tasa de falsos negativos, corresponde al $7 \%$, lo que está de acuerdo con la literatura donde se encuentran falsos negativos del 1.8 al $40 \%(10,13,15,23)$.

Se encuentra en el presente estudio una tasa alta de falsos positivos, $(58 \%)$, que se atribuye al gran número de citologías A3 en las cuales se encontró en la patología una cervicitis.

Es de anotar que en la literatura se encuentra un índice mayor de falsos positivos del $28 \%$ (29).

Se observa que la citología frente al resultado final de patología es menor en el $33 \%$ de los casos.

La frecuencia de citología negativa según las diferentes patologías, corresponde a lo referido en la literatura:

$\begin{array}{lll} & \text { Presente Estudio } & \text { Literatura } \\ \text { NIC I } & 14 \% & 31.6-43.4 \%(1,31) \\ \text { NIC II } & 11 & 15-22 \%(1,31) \\ \text { NIC III } & 4 & 6.2-13.7(1.31)\end{array}$

Es de anotar que a mayor grado de lesión es menor el porcentaje de citología negativa.

Para la citología A2 con atípias severas, se encuentra un $21 \%$ de NIC. Esto se correlaciona con los estudios de Nou- moff y Jones $(36,37)$ que refieren un $25-29 \%$ de NIC en dichas citologías.

La relación entre citología A5 y Ca. invasivo, da una sensibilidad del $56 \%$ con una tasa de falsos negativos del 44\%. Para la citología A4-A5 con Ca. de cervix, da una sensibilidad del $24 \%$ y una tasa de falsos negativos del $76 \%$. Esto se correlaciona con la literatura, donde se describen una tasa de falsos negativos de la citología frente al Ca. de cervix entre el 6 y el 55\% (23).

Por otro lado, encontramos que tanto para la citología A5 como en conjunto A4-A5 la especifidad y valor predictivo negativo son altos. (80-94\% y $98-80 \%)$.

La correlación de la citología frente a la colposcopia nos revela una sensibilidad de la primera del $88 \%$ con un índice de falsos negativos del $12 \%$. Esto se correlaciona con la literatura donde el índice de falsos negativos de la citología frente a la colcoscopia oscila entre 10.3 a $13 \%$ (13).

- Analizando el resultado de la biopsia frente a la pieza final de anatomía patológica, encontramos que esta ofrece una sensibilidad del $91 \%$, con una tasa de falsos negativos del $9 \%$. Esto se correlaciona con varios estudios, donde la sensibilidad se encuentra entre el $84-94 \%(28,32,33,34)$ con un índice de falsos negativos del 0.3 al 34\% $(14,18,30,32)$.

Es de anotar que la biopsia solo refiere una lesión menor que la encontrada en la pieza final en el $15 \%$ de los casos, lo que se correlaciona con la literatura, donde esta cifra oscila entre $1.2 \%$ y $25 \%$ de los casos $(26,27)$.

Desde este punto de vista, la biopsia es un parámetro indirecto de la evolución de la colcoscopia, pues como sabemos estas se toman como biopsias dirigidas bajo colposcopia, y de acuerdo con los resultados encontrados, nos refiere el gran valor que tiene la colposcopia en nuestra institución.

- En cuanto a la frecuencia de colposcopia insatisfactoria, encontramos una frecuencia del $34 \%$, lo que corresponde a lo encontrado en la literatura que va del $4 \%$ al $57 \%$ (ver revisión bibliográfica).

Aunque es un resultado un poco alto, esto se debe a la edad de las pacientes de nuestra clínica, con un $41 \%$ mayores de 40 años, y en las cuales es el porcentaje de colposcopia insatisfactoria es del $52 \%$ (en la literatura se describen tasas hasta del $78 \%$ (3). Además encontramos que en las pacientes menores de 40 años, el porcentaje de colposcopias insatisfactorias solo fue del $21 \%$.

- Esto se demuestra en la Gráfica No. 1, donde observamos que la frecuencia de colposcopia insatisfactoria aumenta con la edad en nuestras pacientes.

En cuanto a los casos con lesión endocervical, se encontró en $67 \%$ de los casos, una colposcopia insatisfactoria.

- Correlacionando la colposcopia con la lesión final de patología, encontramos una sensibilidad del $89 \%$, lo que se correlaciona con la literatura donde se encuentra una correlación entre colposcopia y lesión hitológica del $78 \%$ al $92 \%$ $(31,34)$.

- El índice de falsos negativos encontrado en el presente estudio fue de $11 \%$ para la colposcopia, lo que se interpola con los estudios de la literatura donde oscilan entre $0.5 \%$ al $30 \%(12,30)$.

Aunque los falsos positivos encontrados para la colposcopia en el presente estudio son altos (36\%), encontramos 
un valor predictivo positivo también bastante alto (86\%).

- En cuanto a las lesiones por condiloma, encontramos una sensibilidad de la citologia de solo el $12 \%$, con unos falsos negativos del $88 \%$.

En cuanto a la colposcopia para lesión por condiloma, encontramos una sensibilidad del $63 \%$, con una tasa de falsos negativos del $37 \%$.

Es de anotar que la lesión más frecuente en los casos de condiloma comprobados por patología, fue la leucoplasia (con el 92\% de casos), el seudomosaico y el área papilífera.

- En los casos que la biopsia reveló compromiso glandular, en ninguno se comprobó por la pieza final un compromiso de microinvasión o invasión.

- En cuanto al resultado de la colposcopia, encontramos que la lesión más frecuente en todos los casos fue la leucoplasia con $66 \%$ del total.

La zona de transformación típica se encuentra en el 50\% de los casos y la ectopía en el $32 \%$ de los casos.

El porcentaje de colpitis es de solo $33 \%$ de los casos.

Para la cervicitis, las imágenes más frecuentes encontradas, son la zona de transformación típica (75\%) y el epitelio acetoblanco con $67 \%$.

Para todos los casos de NIC, se establece un patrón colposcópico que corresponde a la leucoplasia (Sensibilidad del $55 \%$ ) y el menor grado el mosaico.

Encontramos que la frecuencia de mosaico aumenta con el mayor grado de compromiso, siendo del $11 \%$ para el NIC I y del $33 \%$ para el microinvasivo y $30 \%$ para el NIC III.

Lo mismo ocurre con el punteado, donde la frecuencia para NIC I es de $23 \%$ y para NIC III es de $67 \%$.

Este patrón de leucoplasia, punteado y mosaico, se correlaciona con las imágenes descritas en la literatura para NIC $(7,9,14,15,24)$.

- No se puede realizar un análisis acerca de las imágenes propias para el microinvasivo, debido al pequeño número de casos.

- En cuanto al patrón colposcópico del carcinoma invasivo, encontramos un predominio de vasos atípicos, así: vasos IV (Sensibilidad 73\%), vasos V (sensibilidad 50\%) y proliferación carcinomatosa (sensibilidad del 65\%).

- Es de anotar que la especificidad de estas imágenes frente al Invasor corresponden a una especificidad del $86 \%$, $98 \%$ y $99 \%$, respectivamente.

- La frecuencia de vasos IV, presenta una frecuencia en ascenso, de acuerdo con el grado de compromiso cervical, teniendo una frecuencia para NIC II del $16 \%$, para NIC III del $24 \%$ y para Ca. Invasivo del $73 \%$.

Los vasos V, aparecen con baja frecuencia en el NIC III (3\%) y realzan su frecuencia en el Ca., invasor con el $50 \%$.

- Estos hallazgos se correlacionan con la literatura, en la cual se observa un predominio de vasos atípicos en las descripciones del $\mathrm{Ca}$. Invasor $(1,2,9,12,14,15,16,24,25)$.

\section{Conclusiones}

1. Basados en la alta sensibilidad de la citología, y del bajo índice de falsos negativos de la misma, podemos concluir que el grado de lesión histológica en nuestras pacientes, se correlaciona directamente con el grado de lesión citológica.

La citología nos ayuda a establecer en nuestra institu- ción, las pacientes que en verdad están enfermas, aunque no nos dice con certeza si la paciente está sana, en caso que dicha citología sea negativa (baja especificidad).

Por otro lado, nos encontramos con una tasa alta de sobrediagnóstico por citología, como no lo demuestra el alto índice de falsos positivos.

2. Ante una paciente con citología A2 con atípias severas, se deben practicar otro tipo de exámenes diagnósticos (colposcopia), pues existe la posibilidad de encontrar un NIC en el $21 \%$ de los casos.

3. Existe un sobrediagnóstico por citología de Carcinoma Invasor, como lo demuestra la tasa de falsos negativos de citología A4-A5. Esto estaría en favor de la citología como Screning, pues en estos casos es mejor pecar por exceso que por defecto.

4. La sensibilidad de la citología en nuestra institución (93\%), se correlaciona bastante bien con la sensibilidad de la colposcopia (89\%) y con la sensibilidad de la biopsia dirigida $(91 \%)$.

Si analizamos estos tres métodos de diagnóstico juntos, llegamos a la conclusión hecha por otros autores, en donde la confiabilidad diagnóstica de los métodos en conjunto, para Neoplasia, oscila entre el $91-100 \%$ $(19,26)$.

5. La alta sensibilidad de la biopsia ( $91 \%)$, nos indica en forma indirecta el gran valor diagnóstico de la colposcopia en nuestra institución, debido a que todas las biopsias analizadas son biopsias bajo colposcopia.

6. La frecuencia de colposcopia insatisfactoria en nuestra institución (34\%), es relativamente alta, debido a la edad avanzada de nuestras pacientes.

7. El porcentaje de colposcopia insatisfactoria, nos revela el grado de lesión endocervical en nuestras pacientes, (67\%), lo que se correlaciona directamente con la alta frecuencia de endopía en todos los casos (11\%).

8. La alta sensibilidad de la colposcopia y su bajo índice de falsos negativos, coloca a nuestros colposcopitas en un alto grado de confiabilidad, comparado con estadísticas de otros países.

9. Aunque el sobrediagnóstico por colposcopia es un tanto alto (falsos positivos), volvemos a recalcar que en dichas pacientes es mejor pecar por exceso, que por defecto.

10. La citología en nuestro instituto, presenta deficiencias notables en el diagnóstico de condiloma. Esto se debe posiblemente a la reciente experiencia y búsqueda de coilocitos en las citologías procesadas. Las bajas pruebas de significancia para la colposcopia en la búsqueda de condiloma, se deben posiblemente a la poca experiencia en este campo.

Basamos la anterior aseveración además, en la infinidad de imágenes y nomenclaturas referidas como imágenes compatibles con condiloma.

En cuanto a lo anterior, se debe establecer en nuestro instituto una nomenclatura concisa para designar a este tipo de lesiones.

Encontramos que la lesión colposcópica más frecuente y característica para condiloma, son el seudomosaico y la zona papilífera con leucoplasia. 
11. El rotulado de compromiso glandular en la biopsia no tiene relación con invasión. Por tanto, en estas pacientes no está justificado realizar cono, si ésta es la única indicación del mismo.

12. Las imágenes colposcópicas para Nic corresponden a Leucoplasia, Base y Mosaico. (Cuadro No. 20). Concluimos que a mayor compromiso (mayor grado de lesión) aparece con mayor frecuencia el punteado y el mosaico.

14. El patrón colposcópico de Carcinoma invasivo corresponde a vasos atípicos IV, V, proliferación carcinomatosa y úlcera. (Cuadro No. 20).

Notamos que los vasos IV aparecen con más frecuencia a mayor compromiso de invasión en la patología.

Estos hallazgos se correlacionan directamente con lo expuesto en la literatura.

\section{Cuadro No. 20 \\ PATRONES COLPOSCOPICOS PARA LAS DIFERENTES PATOLOGIAS EN EL INSTITUTO MATERNO INFANTIL}

\begin{tabular}{|c|c|c|}
\hline Lesión & Patrón colposcópico & \\
\hline $\begin{array}{l}\text { Cervicitis } \\
\text { NICI }\end{array}$ & $\begin{array}{l}\text { ZTT, Epitelio acetoblanco, colpitis a puntos rojos. } \\
\text { Leucop., punteado, vasos III }\end{array}$ & \\
\hline NIC II & Leucop., punteado, vasos III & $\begin{array}{l}\text { Leucoplasia } \\
\text { Punteado } \\
\text { Mosaico }\end{array}$ \\
\hline $\begin{array}{l}\text { NIC III } \\
\text { Microinvasivo } \\
\text { Invasivo }\end{array}$ & $\begin{array}{l}\text { Leucop., punt., vasos IV, mosaico } \\
\text { Leucoplasia, vasos IV } \\
\text { Leucoplasia, punteado } \\
\text { Vasos IV, V, proliferación carcinomatosa } \\
\text { Ulcera. }\end{array}$ & \\
\hline
\end{tabular}

(ZTT: Zona de Transformación típica).

\section{BIBLIOGRAFIA}

1. Wethich DW. Analysis of the factors involved in the colposcipic evaluation of 2194 patients with abnormal papanicolau smears. Am. J. Obstet. Gynecol. 1986; 154:1339

2. Sillman F, Boyce J, Fruchter R. The significance of atypical vessels and neovascularization in cervical neoplasia. Am. J. Obstet. Gynecol. $1981 ; 139: 154$

3. Hatch KD., Shingleton HM., Orr JW., Gore H, Soong S. Role of endocervical curettage in colposcopy. Obstet. Gynecol. 1985; 65:403

4. Moseley KR, Dihn TV, Hannigan EU, Dillard EAjr., Yandell RB. Necessity of endocervical curettage in colposcopy. Am. J. Obstet. Gynecol. 1986; 154:992

5. Spirtos NM, Schlaerth JP, d'Ablaing III G, Morrow C.P, A Critical evaluation of the endocervical curettage. Obstet. Gynecol. 1987; 70:729

6. Benedet JL, Anderson GH. The accuracy of colposcopy in the diagnosis of preclinical invasive squamous carcinoma of the cervix. Colsposco. Gynecol. Laser. Surg. 1987; 3;129

7. Choo YC. Br. J. Obstet. Gynecol. 1984; 9:1156

8. Noda S. Aust. N.Z.J. Obstet. Gynecol. 1981; 21:37

9. Curr. Probl. Cancer. March/April. 1988. Pag 92.

10. Hudson E. Prevención de cáncer cérvico-uterino: Importancia del frotis citológico. Ginecología y obstetricia: Temas actuales. 1985; $1: 41$

11. Coppleson M. Diagnóstico y Tratamiento del Cáncer Cérvico Uterino invasor en etapa incidente (Preclínica). Ginecología y Obstetricia: Temas actuales. 1985; 1:163

12. Shier RM. DePetrillo D. The outpatient management of cervical-intraepithelial neoplasia. Annual clinical conference of cancer. Vol. 29 Gynecologic Cancer: Diagnostic and treatment strategies. University of Texas press, Austin. 1987. Pag. 209.

13. Te linde: Ginecología operatoria. Mattingly D.F., Thompson J.D. Editorial Êl Ateneo S. A. Barcelona. 5a. Edición 1980. Pags. 591 y s.

14. Stafl A, Mattingly RF. Colposcopico diagnosos of cervical neoplasia. Obstet. Gynecol. 1973; 41:168

15. Disais PJ, Creasman WT. Clinical Gynecologic Oncology. 2a. Edic. The C.V. mosby company. 1984. Pags. 15 y s.

16. Zeugyer B. Ginecología infanto juvenil. 2a. Edic. Editorial Médica Panamericana. Junín, Buenos Aires. 1987, Pag. 108

18. Diagnóstico y tratamiento ginecoobstétricos. Ralph C. Benson. Editorial El Manual Moderno S.A. México D.E. 3a. Edición. 1983. 228.

19. Acosta E, Duque JL, Acosta R. Caracterización colposcópica de la cervecitis crónica, la neoplasia intraepitelial cervical y el carcinoma infiltrante. Revista. col. de obstet. ginecol. 1987; 38:399

20. Tovell H, Banogan P, Nash P. Cytology and colpsocopy in the diagnosis and management of preclinical carcioma of the cervix uteri: A learning experience. Am. J. Obstet. Gynecol. 1976; 124:924
21. Matou Aragonés JM. Atlas de colposcopia. Barcelona. Editorial Jima. 1973.

22. Coppleson M, Pixley E, Reid R. 1971. Composcopy springfield Ill: Charles C. Thomas.

23. Fetherson WC. La citología falso-negativa y el Cáncer invasor de cervix. Clin. Obst. Ginecol. 1984; 4:1133

24. Koldstad P. Cambios vasculares en la neoplasia cervical intraepitelial y el Ca. Cervical invasor. Clin. Obstet. Ginecol. 1984; 4:1143

25. Koldstad P., Stafl A. Atlas of colposcopy. 3a. Edic. Oslo: Universitets for laget. 1982.

26. Javaheri G Fejgin MD. Diagnostic value of colposcopic in the investigation of cervical neoplasia. Am J. Obstet. Gynecol. 1990; 137:588

27. Tredway DR, Towsend DE, Houvland DN Upton T.R. Colposcopy and Cryosurgery in cervical intraepithelial neoplasia. Am J. Obstet. Gynecol. 1972; 114:1020

28. Boelter II W.C., Newman R.L. The correlation between colposcopic grading directed punch biopsy and conization. Am. J. Obstet. Gynecol. 1975; 122:945

29. Feldman MJ, Seeve CC, Srebnick E. False positive cervical citology: An important reason for colposcopy. Am J. Obstet Hybecol. 1977; 129:141

30. Crapanzano et. al. Office diagnosis in patients with abnormal cervicovaginal cytosmears: correlation of colposcopic biopsy and citologyc findings. Am. J. Obstet. Gynecol. 1972; 113:967

31. Dresher CHW, Peters III WA, Roberts JA Contribution of endocervical curettage in evaluating abnormal cervical citology. Obstet. Gynecol. 1983; 62:343

32. Towsend DE, Ostergard DR, Mishell DR, Hirose F.M. Abnormal papanicoalu Smears: evaluation by colposcopy biopsies and endocervical curettage. Am J. Obstet. Gynecol. 1970; 108:429

33. Selim MA, So-Bosita JL, Blair OM, Little BA. Cervical Biopsyversus conization. Obstet. Gynecol. 1973; 41:177

34. Ronk DA, Jimerson GJ, Merrill JA. Evaluation of abnormal cervical cytology. Obstet. Gynecol. 1977; 49:581

35. The false negative Smear. II. The trapping effect of collecting instruments. Obstet. Gynecol. 1977; 49:576

36. Noumoff JS Atipya in cervical cytology as a risk factor for intraepithelial Neoplasia. Am J. Obstet. Gynecol. 1987; 156:628

37. Jones DE. et. al. Evaluation of the atypical Pap Smears. Am J. Obstet. Gynecol. 1987; 157:544

38. Piver MS Manual of Gynecologic Oncology and Gynecology. 1a. Edic. Sittle, Brown and Company. 1989. 58.

39. Hamperi H, Kaufman K. The cervix uteri at different Ages. Obstet. Gynecol. 1959; 14:621 